\title{
Knowledge, Perception, and Implementation of Personal Protective Measures by Citizens during the COVID-19 Outbreak in Northern Cyprus: A Cross-Sectional Survey
}

\author{
Azze Buket Yilmaz' (D), Emrah Guler² (D), Buket Baddal ${ }^{2}$ (D) \\ 'Department of Pharmaceutical Microbiology, Near East University Facult of Pharmacy, Nicosia, Cyprus \\ ${ }^{2}$ Department of Medical Microbiology and Clinical Microbiology, Near East University Faculty of Medicine, Nicosia, Cyprus
}

ORCID iDs of the authors: A.B.Y. 0000-0002-3708-0302; E.G. 0000-0002-1635-005I; B.B. 0000-0003-33I9-2I79.

Cite this article as: Yilmaz AB, Guler E, Baddal B. Knowledge, Perception, and Implementation of Personal Protective Measures by Citizens during the COVID-19 Outbreak in Northern Cyprus: A Cross-Sectional Survey. Cyprus J Med Sci. 2021; 6(3): 208-216.

\section{BACKGROUND/AIMS}

Severe acute respiratory syndrome coronavirus 2 has generated over 57 million cases of coronavirus disease 2019 (COVID-19) worldwide and has led to the implementation of strict measures in all countries. The aim of this study was to investigate the knowledge, attitude, and implementation status of personal protective measures (PPMs) by Northern Cyprus citizens and their access to personal protective equipment (PPE) during the COVID-19 outbreak.

\section{MATERIAL and METHODS}

This was a cross-sectional study based on internet-based survey. A total of 406 participants were recruited between May l, 2020 and May 4, 2020 via authors' networks to complete a questionnaire. Participants were asked to indicate how often they implemented five PPMs recommended by the World Health Organization, their daily frequency of hand hygiene events, COVID-19 knowledge, and the availability of PPE in their hometown.

\section{RESULTS}

The prevalence of five PPMs was 65.0-95.6\%, with the highest being hand hygiene and the lowest being avoiding touching the eyes, nose, and mouth. Gloves ( $86.2 \%$ ), surgical masks (52.2\%), and cloth face masks (47.3\%) were the most commonly used PPE in public areas. Majority of responders were aware of the incubation time $(90.4 \%)$ and indirect route of viral transmission $(89.7 \%)$, whereas the knowledge of droplet $(72.2 \%)$ and aerosol transmission $(43.6 \%)$ was lower. Women washed their hands more frequently than men $(P=$ .008), and the 15-54 age group was statistically more knowledgeable about transmission routes compared with $>55$ age group $(P=$ .003). Knowledge was gained mainly through the social media and TV.

\section{CONCLUSION}

Overall, citizens implemented protective measures effectively and were strictly coherent to government-induced curfew and selfisolation measurements with a high public awareness.

Keywords: COVID-19, personal protective equipment, personal protective measures, citizens, Northern Cyprus

\section{INTRODUCTION}

Originated from Wuhan, China, in the late 2019, the severe acute respiratory syndrome coronavirus 2 (SARS-CoV-2), the etiological agent of the coronavirus disease 2019 (COVID-19), has emerged as a significant threat to public health and has been declared as a pandemic by the World Health Organization (WHO), with over 57.8 million cases and I.3 million deaths occurred worldwide as of November 22, 2020.' While SARS-CoV-2 was initially observed in East Asia, Europe consequently became the center of the pandemic, ${ }^{2}$ followed by the United States and Latin America and is currently adversely affecting multiple African countries.

With the rapid surge in the number of people infected with SARS-CoV-2, WHO emphasized the urgent need to coordinate international collaborative efforts to minimize the threat in all affected countries in order to prevent the rapid spread of COVID-19. ${ }^{3}$ While the battle against COVID-19 is ongoing, multiple measures have been taken by governments 
in an effort to slow down and mitigate the viral transmission of the respiratory virus SARS-CoV-2 in the local population, such as closing down schools; banning public gatherings/events; stopping mass movements via land, sea, and airports; imposing partial/full curfew; implementation of the social distance rules; and compulsory use of face masks. When formulating a health policy, key front-line workers within multidisciplinary teams should be a part of the policy planning to ensure that these policies function effectively. Indeed, government health education messages represent a key source of information for citizens for promoting their self-protective practices against respiratory infectious diseases. These preventive messages generally emphasize improved hygiene, face-mask use, and social distancing measures, including avoiding crowds during epidemics. $^{4}$ Although the decision-making and application of such measures by governmental bodies are essential for the control of a pandemic, the level of awareness and adherence of citizens to control measures is also crucial for the successful application of the aforementioned measures.

In the absence of an effective vaccine, public implementation of personal protective measures (PPMs) is fundamental. WHO has recommended five main PPMs against COVID-19: hand hygiene; social distancing measures; avoiding touching the eyes, nose, and mouth; practice of respiratory hygiene; and self-isolation. ${ }^{5}$ While government-imposed social distancing measures alone have been estimated to delay a peak of cases, the combination of this intervention with self-imposed prevention measures such as handwashing and mask-wearing has been shown to further delay a large epidemic and buy time for healthcare systems to prepare for an increasing COVID-19 burden. ${ }^{6}$ In a case-control study investigating the effectiveness of PPMs against SARS-CoV-2 infection including maskwearing, handwashing, and social distancing, the consistent use of protective measures was found to be independently associated with lower risk for SARS-CoV-2 infection in the general public.' Evidence from the literature indicates that fre-

\section{Main Points}

- The level of awareness and adherence of citizens to control measures during the pandemic is crucial for the prevention of COVID-19.

- Majority of North Cyprus citizens were aware of the incubation time for COVID-19 (90.4\%) and indirect route of viral transmission $(89.7 \%)$, whereas the knowledge of droplet $(72.2 \%)$ and aerosol transmission (43.6\%) was lower.

- The prevalence of the application of PPMs among citizens was between 65 and $95.6 \%$, highest being handwashing and lowest being avoiding touching the eyes, nose, and mouth.

- Gloves (86.2\%), surgical masks (52.2\%), and cloth face masks $(47.3 \%)$ were the most commonly used personal protective equipment (PPE) in public areas. Availability of PPE remained high during the pandemic in the country.

- North Cyprus citizens effectively implemented protective measures and were strictly coherent to governmentinduced curfew and self-isolation measurements with a high public awareness. quent hand-washing would reduce viral transmission risk by $55 \%,{ }^{8}$ and epidemiological studies have suggested that handwashing is an effective measure against SARS transmission in health care and community settings." In a separate metaanalysis, regular hand hygiene was detected to have a protective effect against the 2009 influenza pandemic and was recommended as an effective measure to limit the transmission of pandemics. $^{10}$ Indeed, recent studies indicate that wearing masks, hand hygiene, and social distancing not only contribute to the prevention of COVID-19 but also lead to the decline of other respiratory infectious diseases such as influenza, enterovirus, and all-cause pneumonia." Mask-wearing and instant hand hygiene together have been proposed to slow down the exponential spread of SARS-CoV-2. ${ }^{12}$ While considered an important factor for reducing viral transmission, the incorrect use of face masks has also been reported during the COVID-19 pandemic, and compliance rates have been shown to be low in men and persons with low household incomes. ${ }^{13}$ Interestingly, a cross-sectional study revealed that mask wearing was associated with a reduction in face-touching behaviors, particularly touching the nose, mouth, and eyes. ${ }^{14}$

Another recommendation by WHO suggests that citizens should follow advice given by their healthcare provider or their national and local public health authorities in order to obtain information from a reliable source. The level of public adherence to control measures is affected by their knowledge, attitudes, and practices toward COVID-19, and it directly impacts the trajectory of the outbreak in a country. This is particularly valid during outbreaks, which, due to their evolving nature and inherent scientific uncertainties, can be associated with considerable fear in the general public, especially if illness or death rates are high. This was a phenomenon observed during the SARS outbreak in 2003, where studies conducted suggest that the level of panic emotions in the population is directly associated with their knowledge and attitudes toward the infectious disease. ${ }^{15}$

The first case of COVID-19 in Northern Cyprus was reported on March 9, 2020.16 Consequent to the identification of the first case, a wide range of measures have been implemented by the government in order to prevent the spread of the virus. All schools and nonessential workplaces were immediately shut down; country borders were closed to all incoming noncitizen travelers, which was reopened on July I with the condition of 10 days of quarantine. Within the COVID-19 restrictions, large gatherings were banned, a full curfew from 21:00 PM to 06:00 AM was temporarily enacted, and mandatory use of face masks in public areas was implemented. As of November 24, 2020, a total of 1,062 cases and five COVID-19-related deaths have been reported in the country. ${ }^{17}$ In order to facilitate outbreak management of COVID-19 in Northern Cyprus, there is an urgent need to clarify the public awareness of COVID-19 and their status of the implementation of the protective measures, as well as their access to personal protective equipment (PPE) during the COVID-19 pandemic. The aim of this study is to investigate the knowledge, attitude, and practices of Cypriot citizens toward COVID-19 and to measure their coherence to PPMs during the public health crisis.

\section{MATERIAL and METHODS Ethical Approval}

This project was approved by Near East University Institutional Review Board, Project No: YDU/2020/80-III3. Respondent's 
anonymity and confidentiality were ensured. The submission of the answered survey was considered as a consent to participate in the study.

\section{Participants and Data Collection}

This was a cross-sectional study conducted through an internet-based survey between May I and May 4, 2020. Partial curfew from 06:00 to 21:00 in Northern Cyprus began on April I, 2020 and ended on May 4, 2020, while the implementation of the mandatory mask use in public areas began on April 24, 2020. Therefore, the survey dates represent a reliable time period for data collection on self-isolation and coherence to COVID-19 measures. As of May I, the total number of reported COVID-19 cases in Northern Cyprus was 108. ${ }^{18}$ A total of 406 citizens were randomly included in this study. Participants were recruited via authors' networks with local citizens living in Northern Cyprus. A recruitment poster with a link to the questionnaire was posted to online pages through the local COVID19-related media as well as Facebook, emails, and WhatsApp, which contained information on the background, objective, voluntary nature of participation, indications of how to fill in the questionnaire, and the declarations of anonymity and confidentiality of participants. Citizens who indicated that they were unable to adhere to curfew measures due to the essentiality of their jobs (eg, fire brigade and policemen) were excluded from the study.

\section{Measurement}

Survey: The survey consisted two sections and 26 questions. Section I contained eight questions on demographic data, followed by 14 questions regarding the participants' knowledge and attitudes toward PPMs and COVID-19 in section 2. The survey was created by the researchers, and a piloting was performed before the survey was distributed.

Assessment of Sociodemographic Characteristics: The first part of the questionnaire consisted of the assessment of sociodemographic attributes, in which participants responded to demographic variables including gender, age, education status, occupation, current district of residency, smoking (smoker/nonsmoker), chronic disease (present/nonpresent), and seasonal influenza vaccination history (vaccinated/nonvaccinated).

Assessment of Knowledge on COVID-19: Participants were asked to answer questions regarding the transmission routes of SARS-CoV-2 among choices, including droplet, aerosol, and indirect transmission (touching contaminated surfaces and touching eyes, mouth, and nose) routes or indicate if they did not know the transmission route of infection. Participants also responded to a question on the incubation period for SARSCoV-2. Among the choices I-2 days, 2-4 days, 2-14 days, 27 days, and "I do not know the incubation period" were present.

Assesment of WHO Recommended Personal Protective Control Measures: Participants were asked to indicate which of the personal protective control measures including hand hygiene; social distance; avoiding touching nose, mouth, and eyes; and use of face mask they implemented during the pandemic and the frequency of implementation for each measure. For each option, the scale was designed as always, sometimes, rarely, and never. Participants also responded to a question which assessed their coherence to the curfew imposed by the local government, outside of their working hours, with a scale of always, generally, rarely, and never.

The application of PPE by ordinary citizens was evaluated. Individuals were asked to indicate which PPE they used in public areas such as supermarkets, with choices including gloves, surgical masks, vented masks, cloth masks, wrap/scarf, face shield, goggles, and "I do not use any PPE." Participants were also assessed if they applied hand hygiene before and after the PPE use and if they adhered to social-distancing rules while wearing face masks.

Assessment of Hand Hygiene Events per Day: Questions were designed to evaluate the hand hygiene measures applied by citizens. Individuals were initially asked which products they use when they apply hand hygiene and selected from various options including water, soap and water, alcohol-based hand sanitizer, pure alcohol, cologne with at least $70 \%$ alcohol content, wet tissues, or "I do not use any products." The number of hand hygiene events per day was also evaluated. Participants reported the mean number hand hygiene events based on soap and water and alcohol-based hand sanitizers per day.

Assessment of The Availability of PPE During The Pandemic: As the use of PPE and application of hand sanitation can be affected by their availability in a local town or city, participants were asked to report their access to these products during the pandemic. Participants responded to questions for the availability of masks, gloves, and hand sanitizers with a scale of always, generally, rarely, and never for each product.

Assessment of The Attitude Toward Healthcare Facilities During The Pandemic: The citizens were asked if, during the pandemic, they felt any reservation from visiting hospitals or any healthcare facility where they responded as yes or no. Additionally, participants were asked if they would go for a COVID-19 test in a mobile test center in a hospital carpark without leaving their car for which they responded as yes or no.

Assessment on The Source of COVID-19-Related Information: In this survey, the use of reliable source of personal protective procedures and the source of COVID-19-related updates by participants were evaluated. Individuals were asked to choose among WHO, Turkish Republic of Northern Cyprus Ministry of Health, Turkish Republic of Northern Cyprus Medical Association, newspapers, TV, and social media for the most commonly used source of personal protective procedures. Citizens also indicated the most commonly used sources of COVID-19-related updates among scientific articles, TV, radio, internet sites, newspapers, brochures/flyers, social media, family/friends/relatives, and WhatsApp group.

\section{Statistical Analysis}

Statistical analyses were performed using Statistical Package for the Social Sciences (SPSS) version 23 (IBM SPSS Corp; Armonk, NY, USA). Knowledge and attitudes and practices of different individuals according to demographic characteristics were compared using independent samples t test or Chisquare test as appropriate. Binary logistic regression analysis using demographic variables as independent variables and knowledge or practices as the outcome variable were conducted to identify factors associated with attitudes and practices. $P$ value of .05 or less was considered as significant. 


\section{TABLE I. Demographic Characteristics of Participants}

\begin{tabular}{|lcc}
$\begin{array}{l}\text { Participant } \\
\text { characteristics }\end{array}$ & Male & $\begin{array}{c}\text { Number of } \\
\text { participants, } \mathbf{n}(\%)\end{array}$ \\
\hline Gender & Female & $191(47)$ \\
Age group (years) & $15-20$ & $215(53)$ \\
& $21-34$ & $8(2)$ \\
& $35-44$ & $151(37.2)$ \\
& $45-54$ & $99(24.4)$ \\
Residential area & $55-64$ & $38(9.4)$ \\
& $65+$ & $86(21.2)$ \\
& Kyrenia & $24(5.9)$ \\
& Nicosia & $71(17.5)$ \\
& Famagusta & $210(51.7)$ \\
Education & Trikomo & $63(15.5)$ \\
& Morphou & $16(3.9)$ \\
& Lefka & $20(4.9)$ \\
& Primary school & $26(6.4)$ \\
Smoking & Secondary school & $9(2.2)$ \\
& High school & $22(5.4)$ \\
Chronic disease & Bachelor's degree & $72(17.7)$ \\
& Master's degree or above & $202(49.8)$ \\
Seasonal influenza & Smoker & $116(24.9)$ \\
vaccination & Nonsmoker & $290(71.4)$ \\
& Yes & $68(16.7)$ \\
& No & $338(83.3)$ \\
& Yes & $26(6.4)$ \\
& No & $380(93.6)$ \\
& &
\end{tabular}

\section{RESULTS}

A total of 406 participants (53\% females) completed the survey questionnaire. The demographic characteristics of participants are shown in Table I. Participants belonged to a broad distribution of age range, residential areas across the country, and educational backgrounds. A large majority of the responders had a Bachelor's degree (49.8\%) or higher (24.9\%), $71.4 \%$ were nonsmokers, and $83.3 \%$ did not have any chronic disease.

Before the assessment of the implementation of PPMs by citizens, participants were asked two questions regarding the transmission routes and incubation time of COVID-19 in order to measure their level of knowledge of the disease, which may also affect their inclination to properly apply protective measures and PPE use. A striking $89.7 \%$ of the individuals responded with indirect transmission route for the disease, whereas 72.2 and $43.6 \%$ were aware of droplet and aerosol transmission routes, respectively (Figure la). There were no statistical differences between men and women in terms of their knowledge of all the three disease transmission routes ( $P=$ .089). When the age groups and their knowledge on transmission routes were analyzed, a statistically significant association was found in the age group 15-34 and 34-54 compared with the 55 and above $(P=.046)$. This suggests that younger citizens were more knowledgeable about disease transmission compared with the elderly. Regarding the incubation time for COVID-19, a vast majority (90.4\%) were aware of the 2-14 days of incubation time (Figure lb). There was no statistically significant association between gender or age groups in terms of knowledge of disease incubation time. Binary logistic regression analysis showed that education levels of individuals and COVID-19 incubation time knowledge were not significantly associated ( $P=.915$ ), suggesting that individuals at all education levels were knowledgeable about COVID-19, and that this knowledge was gained through educatory channels during the pandemic. However, there was a statistically significant difference between education level and transmission routes of the disease $(P=.000)$, in which individuals with a bachelor degree or above had better knowledge and had a higher frequency of correct answers (Table 2). There were no statistical differences between individuals from different cities in terms of their knowledge of the incubation time $(P=.997)$ or the transmission routes of COVID-19 $(P=.427)$.

Figure 2a shows the prevalence of the application of WHO recommended PPMs against COVID-19 by the citizens. Data suggest that participants always implemented hand hygiene and social distancing in public areas with a prevalence of 96.5 and $82.6 \%$, respectively, during the pandemic. Alternatively, 72.6 and $65.8 \%$ of individuals always implemented the use of face masks and avoided touching the nose, mouth, and eyes, respectively. Among all PPMs, hand washing was statistically the most applied measure $(P=.00 \mathrm{l})$. In terms of PPE use, the mostly used equipment was gloves ( $86.2 \%)$, followed by face masks ( $52.2 \%$ surgical masks and $47.3 \%$ cloth masks). Types of

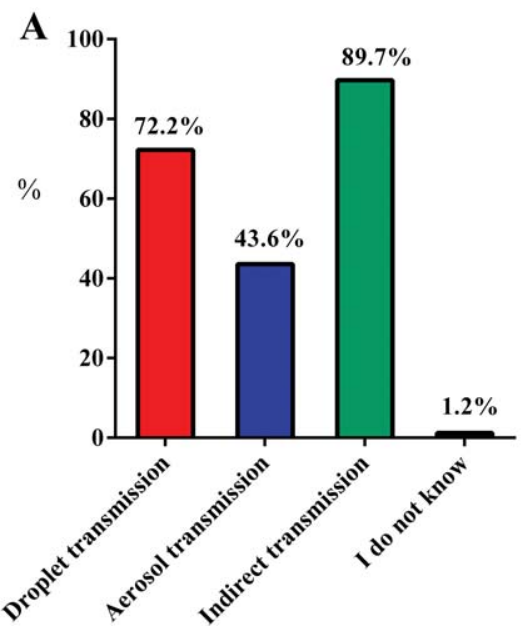

B

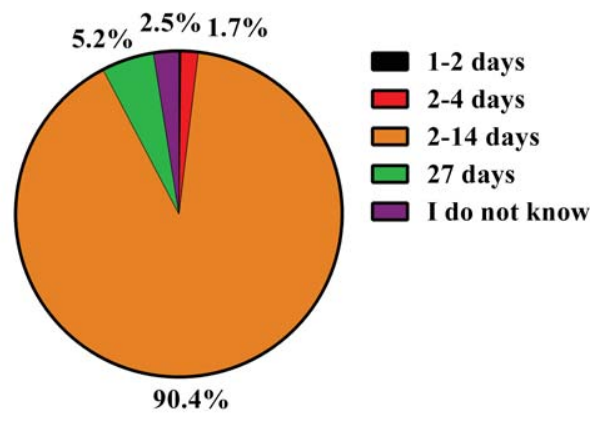

Figure I. a,b. Participant knowledge on COVID-19 characteristics. (a) Participant knowledge on COVID-19 transmission routes (b) Participant knowledge on COVID-19 incubation time 


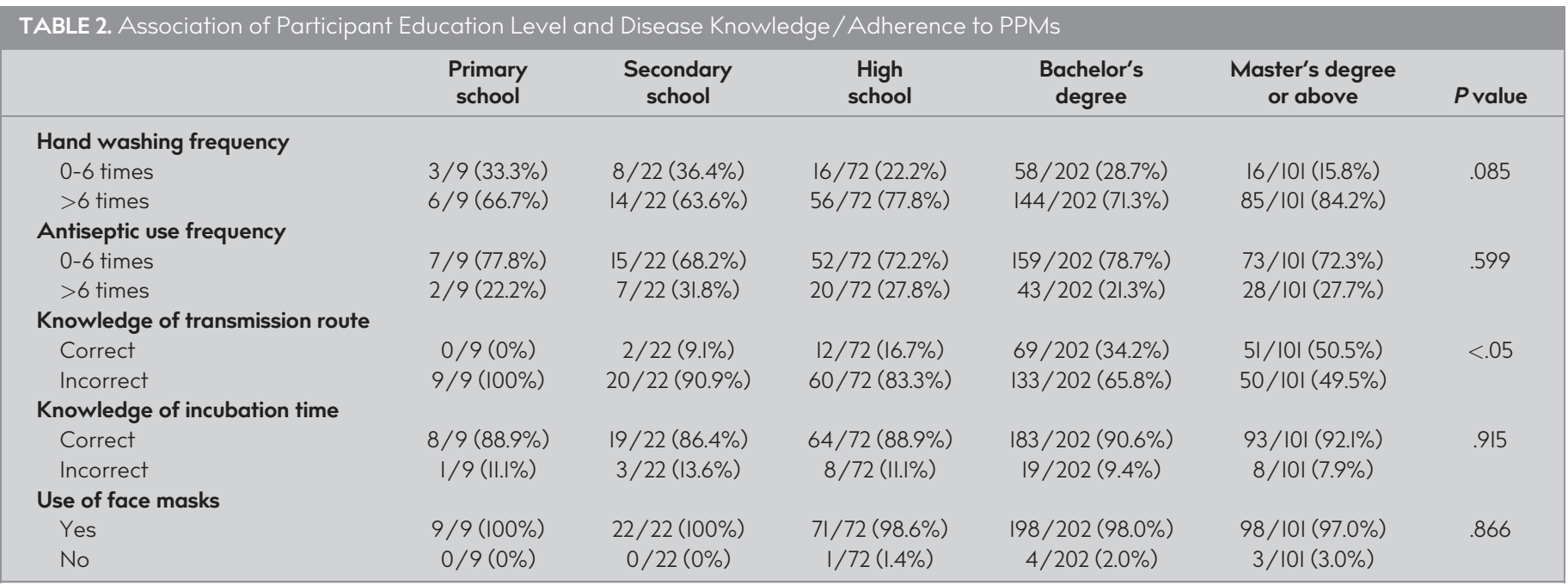

A

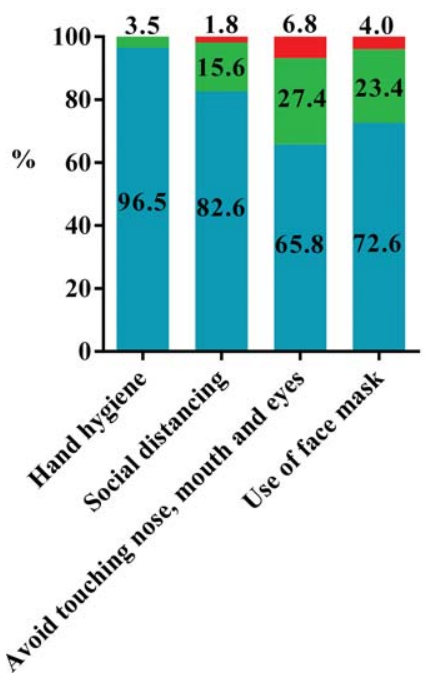

B

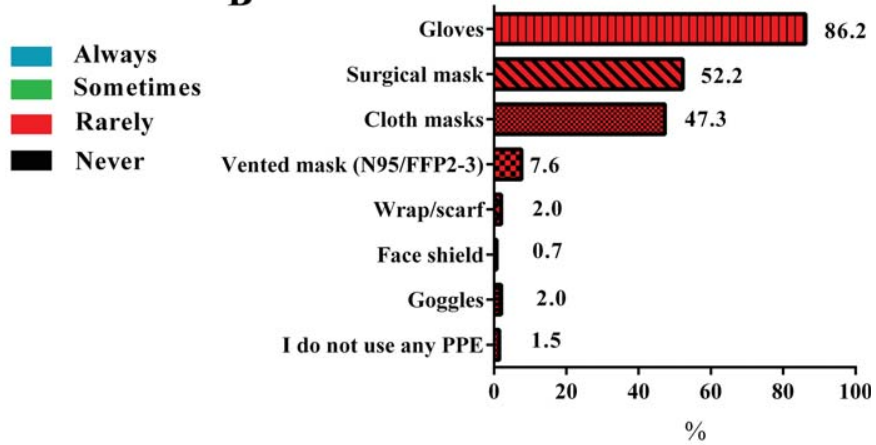

Figure 2. a,b. (a) Application frequency of personal protective measures recommended by WHO, (b) Use of personal protective equipment by participants

TABLE 3. Participant Coherence to Curfew Imposed by the Local Government

\begin{tabular}{|lcccc|} 
& Always, $n(\%)$ & Usually, $n(\%)$ & Rarely, $n(\%)$ & Never, $n(\%)$ \\
\hline Coherence to curfew-self-isolation measures & $323(79.5)$ & $79(19.5)$ & $2(0.5)$
\end{tabular}

PPE used by participants and their usage prevalence are shown in Figure 2b. When participants from different cities were evaluated in terms of the use of masks in public areas, the prevalence of citizens who applied all types of masks was found to be $94.4-100 \%$ in all cities with no statistical differences among them $(P=.084)$. Similarly, no statistical association was found between education level and the use of masks $(P=.866)$ (Table 2).

Of all the participants, adherence to hand hygiene before and after mask and glove use was 90.1 and $93.8 \%$, respectively, indicated to practice $2-m$ social distancing while in public places using masks. Overall, citizens were coherent to curfew imposed by the local government (Table 3).

When participants were asked to indicate which hand hygiene products they used, majority indicated to use soap and water (95.6\%), followed by hand sanitizer ( $81 \%$ ) and cologne with at least $70 \%$ alcohol content (69\%) (Figure 3a). Figure $3 b$ and $c$ shows the total number of hand hygiene events per day. A high proportion of citizens $(47.3 \%)$ responded to wash their hands more than 10 times a day, while a high prevalence was observed for the application of hand sanitizer l-3 times a day (37.9\%) (Figure 3c). There was a statistically significant 
A

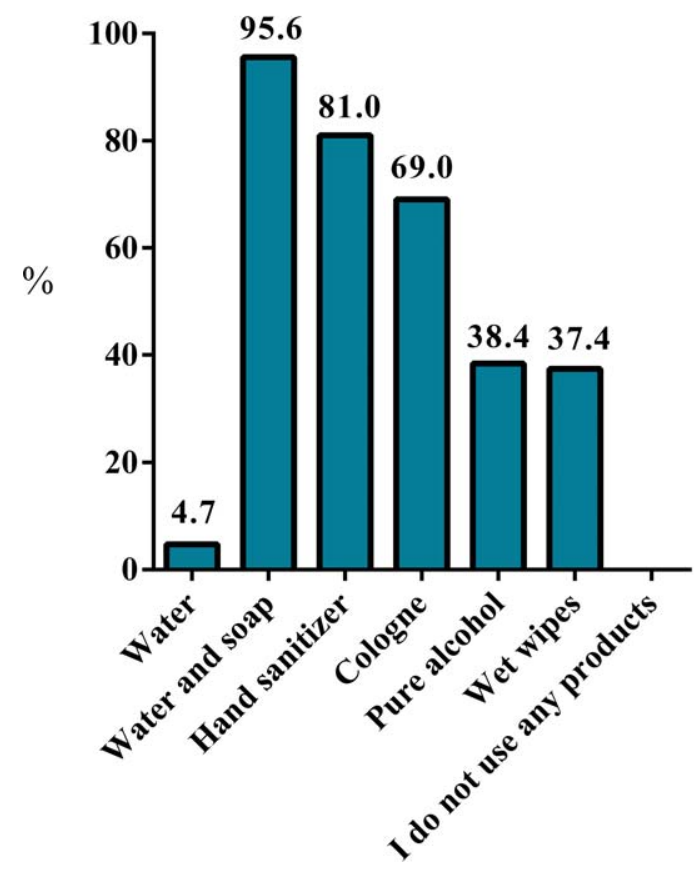

B

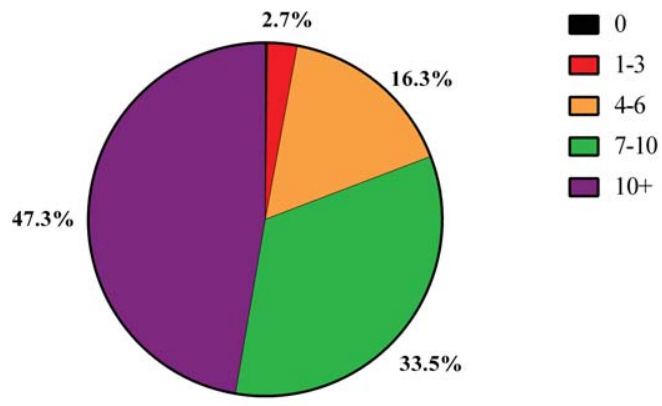

C

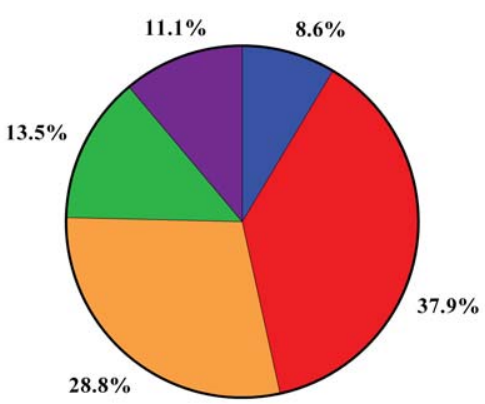

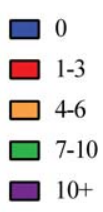

Figure 3. a-c. (a) The proportion of participants applying hand hygiene products, (b) Frequency of daily hand washing events, (c) Frequency of daily hand sanitation events

difference between age groups and hand washing frequency $(P=.046)$, where individuals in age group 35-54 had a higher hand washing frequency (7 or more) compared with other groups. On the contrary, there was no statistically significant difference between age groups and frequency of hand sanitation application $(P=.159)$. Interestingly, when the gender of the participants was analyzed, a statistically significant difference was observed in terms of hand-washing frequency, where women washed their hands ( $>6$ times a day) more frequently than men $(P=0.008)$. There was no statistically significant association between gender and application of hand sanitizers $(P=.652)$. In terms of demographics, our analysis showed that the distribution of individuals who had a hand washing frequency $>6$ times a day was $87.5 \%$ in Trikomo, $79.5 \%$ in Nicosia, $75.0 \%$ in Morphou, $70.4 \%$ in Kyrenia, $66.7 \%$ in Famagusta, and $65.4 \%$ in Lefka. Similarly, the distribution of antiseptic use frequency $>6$ times a day was $31.3 \%$ in Trikomo, 30.8\% in Lefka, $28.2 \%$ in Kyrenia, $25.4 \%$ in Famagusta, $22.4 \%$ in Nicosia, and $20.0 \%$ in Morphou. There were no statistically significant differences between different cities in terms of hand hygiene events as shown in Table 4 (hand washing, $P=.153$; antiseptic use, $P=$ 820).

When the local availability of PPE for the participants was assessed, masks, gloves, and hand sanitizer were found accessible to public use at a large extend. However, although less than $25 \%$, some of the participants were rarely or never able to find these PPE products available at the pharmacies or supermarkets during the pandemic (Figure 4).

Majority of citizens (82\%) indicated that during the pandemic, they felt reservation from visiting hospitals or healthcare facilities. Additionally, $84.2 \%$ of the participants responded that they would be comfortable with going for a COVID-19 test in a mobile test center in a hospital carpark without leaving their car. Binary logistic regression analysis showed that education levels of individuals and having a COVID-19 test in a mobile test center were not significantly associated $(P=.266)$,

\begin{tabular}{|c|c|c|c|}
\hline & $0-6$ times & $>6$ times & $P$ value \\
\hline \multicolumn{4}{|c|}{ Hand washing frequency } \\
\hline $15-34$ & $47 / 159(29.6 \%)$ & $112 / 159(70.4 \%)$ & \multirow[t]{3}{*}{$<.05$} \\
\hline $35-54$ & $24 / 137(17.5 \%)$ & $113 / 137(82.5 \%)$ & \\
\hline$>55$ & $30 / 110(23.7 \%)$ & $80 / 110(72.5 \%)$ & \\
\hline Male & $59 / 191(30.9 \%)$ & $132 / 19 \mid(69.1 \%)$ & \multirow[t]{2}{*}{$<.01$} \\
\hline Female & $42 / 215(19.5 \%)$ & $173 / 215(80.5 \%)$ & \\
\hline Kyrenia & $21 / 71(29.6 \%)$ & $50 / 7 \mathrm{l}(70.4 \%)$ & \multirow{6}{*}{.153} \\
\hline Nicosia & $43 / 210(20.5 \%)$ & $167 / 210(79.5 \%)$ & \\
\hline Famagusta & $21 / 63(33.3 \%)$ & $42 / 63(66.7 \%)$ & \\
\hline Trikomo & $2 / 16(12.5 \%)$ & $14 / 16(87.5 \%)$ & \\
\hline Morphou & $5 / 20(25.0 \%)$ & $15 / 20(75.0 \%)$ & \\
\hline Lefka & $9 / 26(34.6 \%)$ & $17 / 26(65.4 \%)$ & \\
\hline \multicolumn{4}{|c|}{ Antiseptic use frequency } \\
\hline $15-34$ & $114 / 159(71.7 \%)$ & $45 / 159(28.3 \%)$ & \multirow[b]{3}{*}{.159} \\
\hline $35-54$ & $102 / 137(74.5 \%)$ & $35 / 137(25.5 \%)$ & \\
\hline$>55$ & $90 / 110(81.8 \%)$ & $20 / 110(18.2 \%)$ & \\
\hline Male & $|42 / 19|(74.3 \%)$ & $49 / 191(25.7 \%)$ & \multirow{2}{*}{.652} \\
\hline Female & $164 / 215(76.3 \%)$ & $51 / 215(23.7 \%)$ & \\
\hline Kyrenia & $51 / 71$ (7I.8\%) & $20 / 71(28.2 \%)$ & \multirow{6}{*}{.820} \\
\hline Nicosia & $163 / 210(77.6 \%)$ & $47 / 210(22.4 \%)$ & \\
\hline Famagusta & $47 / 63(74.6 \%)$ & $16 / 63(25.4 \%)$ & \\
\hline Trikomo & $11 / 16(68.8 \%)$ & $5 / 16(31.3 \%)$ & \\
\hline Morphou & $16 / 20(80.0 \%)$ & $4 / 20(20.0 \%)$ & \\
\hline Lefka & $18 / 26(69.2 \%)$ & $8 / 26(30.8 \%)$ & \\
\hline
\end{tabular}




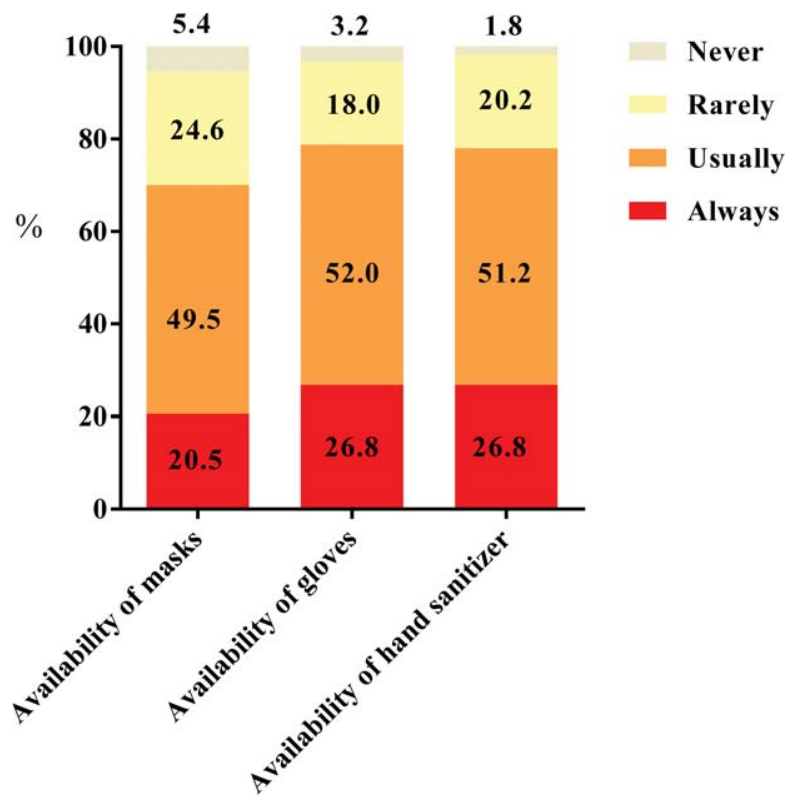

Figure 4. The prevalence of personal protective equipment availability

suggesting that individuals at all education levels were comfortable with having a COVID-19 test in a mobile test center.

Based on the responses, the mostly used source of COVID-19related information was social media (7l.4\%) and TV (70.9\%), while only a minority of citizens used newspapers, scientific articles, and brochures/flyers. The same pattern was also observed for information on personal protective procedures (social media $73.6 \%$ and TV 55.2\%), followed by more reliable sources such as WHO, T.R.N.C. Ministry of Health, and T.R.N.C. Medical Association used at a lesser extend (Table 5).

\section{DISCUSSION}

In the course of a pandemic, the implementation of PPMs represents an important factor for the control of the outbreak and has been shown to have significant protective effects associated. $^{10}$ This study was designed to assess the knowledge, attitudes, and implementation status of PPMs by the citizens in Northern Cyprus between May I and May 4, 2020. The survey period covers an important time period amid COVID-19 pandemic in which all preventive measures enforced by the local government in Northern Cyprus were in place, and the curfew was still ongoing and, therefore, provides a timely assessment. Overall, $70-90 \%$ of the individuals surveyed were knowledgeable about the droplet and indirect transmission routes of disease transmission, whereas the citizens were aware of the more recently described aerosol route of transmission ${ }^{19}$ at a lesser extent, approximately 44\%. The survey responders also demonstrated a $90 \%$ knowledge of the incubation period for COVID-19, demonstrating a high comprehension of disease characteristics during the pandemic. In a different study conducted among in Cyprus, healthcare workers were found to have a satisfactory level of knowledge of the virus. ${ }^{20}$ Interestingly, in our study, younger citizens were statistically more knowledgeable about the disease transmission routes compared with the elderly population above age 55. Recent sur-

$\begin{array}{lc}\text { TABLE 5. The Source of COVID-19-Related } & \text { Information and } \\ \text { Personal Protective Measures } & \mathbf{n}(\%) \\ \text { Source of personal protective procedures } & 299(73.6) \\ \text { Social media } & 224(55.2) \\ \text { TV } & 195(48.0) \\ \text { T.R.N.C. Ministry of Health } & 174(42.9) \\ \text { World Health Organization } & 102(25.1) \\ \text { T.R.N.C Medical Association } & 21(5.2) \\ \text { Newspaper } & \mathbf{n}(\%) \\ \text { Source of COVID-I9 information } & 290(71.4) \\ \text { Social media } & 288(70.9) \\ \text { TV } & 278(68.5) \\ \text { Internet sites } & 83(20.4) \\ \text { Scientific articles } & 40(9.9) \\ \text { Newspaper } & 40(9.9) \\ \text { Family/friends/relatives } & 23(5.7) \\ \text { WhatsApp group } & 16(3.9) \\ \text { Radio } & 4(\mathrm{I}) \\ \text { Brochures/flyers } & \end{array}$

veys on public knowledge on COVID-19 in China, where the pandemic has emerged, demonstrated an overall $90 \%$ correct rate on a COVID-19 knowledge test, which included questions on main clinical symptoms, viral transmission, observation period, and age groups affected. ${ }^{21}$ In a separate study, where the responders were asked question regarding the origin, common signs and symptoms, sources of infection of COVID-19, and awareness of any other pandemic viral infection, the knowledge level of individuals varied according to the profession. ${ }^{22}$ Cross-sectional studies from various countries have showed that there are regional disparities in the attitude toward preventive measures and knowledge of COVID-19.23-27

WHO and its recommendation of five PPMs against COVID-19, namely, hand hygiene; social distancing measures; avoiding touching the eyes, nose, and mouth; use of face masks; and self-isolation, have been taken as a reference by all countries and were communicated to the citizens via local health authorities in each country. Of these measures, the prevalence of hand hygiene and social distancing was the highest and respiratory etiquette was high, whereas the prevalence of avoiding touching nose, mouth, and eyes was the lowest among Northern Cyprus citizens. Interestingly, our study results indicated that women statistically washed their hands more frequently than men. Compared with a recent a study conducted in Japan, with hand hygiene being the highest with $83.8 \%$ and the lowest being avoiding touching eyes, nose, and mouth with $59.8 \%$, the prevalence of PPM application was overall higher in Northern Cyprus. ${ }^{28}$ Similarly, in a population survey performed in the United Kingdom with the age group of $18+$ individuals, the prevalence of respondents taking the aforementioned measures to protect themselves and others from COVID-19 was comparably much lower with face mask implementation being as low as $3 \%$ and hand washing being highest with $83 \%{ }^{29}$ Alternatively, in a study conducted in Hong Kong, enhanced personal hygiene practices were adopted by more than $77 \%$ of citizens. ${ }^{30}$ Medical students, on the other hand, have been shown to adopt social isolation strategies, regular hand washing, and enhanced personal hygiene measures at a rate of more than $80 \%{ }^{31}$ To a surprising extend, Machida et al. ${ }^{32}$ reported a significant improvement in the implementation of PPMs and social distancing measures in particular, during the 
community transmission phase compared to the early phase of the COVID-19 outbreak.

The recommendation on the use of face masks by citizens in public areas has been controversial. In a guidance report dated January 29, 2020, the WHO recommendation was for only individuals with respiratory symptoms to wear a medical mask to avoid unnecessary cost, procurement burden, and false sense of security. ${ }^{33} \mathrm{WHO}$ additionally published interim guidance report on the rational use of PPE for COVID-19, which recommended the use of vented masks (respirators) only for frontline healthcare workers in order to optimize the PPE availability. ${ }^{34}$ However, health authorities in parts of Asia encouraged all citizens to wear masks in public setting, and the Czech Republic applied mandatory use of face masks to prevent viral spread in the community. ${ }^{35}$ The Centers for Disease Prevention and Control has also recommended the use of cloth face coverings or cloth masks in public settings with particular risk of communitybased transmission. ${ }^{36}$ In Northern Cyprus, the mandatory use of face masks was implemented by the local government on April 24, 2020. In the Cypriot community, the practice of surgical mask and cloths mask use was $47-52 \%$ and approximately $8 \%$ for vented masks, indicating a good adherence to government measures against COVID-19 with some room for improvement. In our study, there were no statistical associations between educational level or geographical regions and the use of face masks. In an online survey conducted in China where the pandemic began, $96.9 \%$ of the participants indicated that they use a mask when going to the hospital. ${ }^{37}$ In Japan, the prevalence of wearing masks was $80.9 \% .^{13}$ Indeed, government mandates for face mask use in community has been demonstrated to be associated with a decline in the daily COVID-19 growth rate in the United States. ${ }^{38}$

During the early phase of the pandemic, a major demand of face masks, gloves, and hygiene products arose mainly due to the panic caused by the outbreak, and it has led to the exhaustion of local hygiene products and PPE as well as a steep rise in prices in many countries. ${ }^{39}$ A recent study has indicated a correlation between the spread of COVID-19 and the search for PPE and hand hygiene, which can, to a certain extent, show people's concerns, behaviors, and reactions to sanitary problems and protection recommendations. ${ }^{40}$ In Northern Cyprus, the shortage of gloves, face masks, and hand sanitizers was reported by $21-30 \%$ of the citizen, who indicated they could never find certain PPE types available. In terms of the source of information used by people to gather knowledge about COVID-19, some studies suggested that social media and internet are among the most commonly used sources, ${ }^{29,41}$ whereas people also had a tendency of acquiring information and advice from TV and local health authorities which they expressed as more reliable. ${ }^{30}$ The Cypriot citizens' choice of COVID-19 information and advice on PPMs were similar to previous studies with TV, social media, and local health authorities such as Ministry of Health and Medical Association being represented on top of the list.

As the current study was based on an online survey, there are certain limitations. There can be differences among participants in terms of their understanding and interpretation of the questions. Furthermore, due to the collection of data at a single point in time with the survey, it is difficult to measure changes in the population over time, for instance, from the beginning of outbreak through the pandemic.

\section{CONCLUSION}

In conclusion, the knowledge of the disease characteristics, the practice of PPMs, and coherence to government-induced protective measures were high in Cyprus citizens. This is believed to have advantageous effects on the control of the outbreak in the country.

Ethics Committee Approval: Ethical committee approval was received from the Near East University (YDU/2020/80-III3).

Informed Consent: Written informed consent was obtained from all participants who participated in this study.

Peer-review: Externally peer-reviewed.

Author Contributions: Concept - B.B.; Design - B.B., A.B.Y.; Supervision B.B.; Data Collection and / or Processing - A.B.Y; Analysis and / or Interpretation - E.G., B.B.; Literature Search - A.B.Y., B.B.; Writing Manuscript - A.B.Y., B.B., E.G., Critical Review - B.B.

Acknowledgments: The authors would like to thank all study participants who have provided us with their valuable information and time to accomplish the web-based survey.

Conflict of Interest: The authors have no conflicts of interest to declare.

Financial Disclosure: The authors declared that this study has received no financial support.

\section{REFERENCES}

I. World Health Organization. COVID-19 weekly epidemiological update. 2020. Available at https://www.who.int/publications/ $\mathrm{m}$ /item/weekly-epidemiological-update-24-november-2020. Accessed date: 30 November 2020

2. World Health Organization. WHO announces COVID-19 outbreak a pandemic. 2020. Available at http://www.euro.who.int/en/ health-topics/health-emergencies/coronavirus-covid-19/news/ news/2020/3/who-announces-covid-19-outbreak-a-pandemic. Accessed date: 15 April 2020.

3. World Health Organization. 2019-nCoV outbreak is an emergency of international concern. 2020. Available at http://www.euro. who.int/en/health-topics/health-emergencies/internationalhealth-regulations/news/news/2020/2/2019-ncov-outbreak-isan-emergency-of-international-concern. Accessed date: 8 August 2020.

4. European Centre for Disease Prevention and Control, MERS-CoV worldwide overview-Situation update. 2020. Available at https: / / www.ecdc.europa.eu/en/middle-east-respiratory-syndrome-coronavirus-mers-cov-situation-update. Accessed date: 3 July 2020.

5. World Health Organization. Coronavirus disease (COVID-19) advice for the public. 2020. Available at https://www.who.int/ emergencies/diseases/novel-coronavirus-2019/advice-for-public. Accessed date: 8 August 2020.

6. Teslya A, Pham TM, Godijk NG, Kretzschmar ME, Bootsma MCJ, Rozhnova G. Impact of self-imposed prevention measures and short-term government-imposed social distancing on mitigating and delaying a COVID-19 epidemic: A modelling study. PLoS Med. 2020;17(12):el003499. [CrossRef]

7. Doung-Ngern P, Suphanchaimat R, Panjangampatthana A, Janekrongtham C, Ruampoom D, Daochaeng N, et al. Case-Control Study of Use of Personal Protective Measures and Risk for Severe Acute Respiratory Syndrome Coronavirus 2 Infection, Thailand. Emerg Infect Dis. 2020. [CrossRef] 
8. Jefferson T, Del Mar C, Dooley L, et al. Physical interventions to interrupt or reduce the spread of respiratory viruses: Systematic review. BMJ. 2009;339:B3675. [CrossRef]

9. Fung $\mathrm{ICH}$, Cairncross S. Effectiveness of handwashing in preventing SARS: A review. Trop Med Int Health. 2006;1I:1749-1758. [CrossRef]

10. Saunders-Hastings P, Crispo JAG, Sikora L, Krewski D. Effectiveness of personal protective measures in reducing pandemic influenza transmission: A systematic review and meta-analysis. Epidemics. 2017;20:I-20. [CrossRef]

II. Chiu NC, Chi H, Tai $\mathrm{YL}$, et al. Impact of wearing masks, hand hygiene, and social distancing on influenza, enterovirus, and allcause pneumonia during the coronavirus pandemic: Retrospective national epidemiological surveillance study. J Med Internet Res. 2020;22:E21257. [CrossRef]

12. Ma QX, Shan H, Zhang HL, Li GM, Yang RM, Chen JM. Potential utilities of mask-wearing and instant hand hygiene for fighting SARS-CoV-2. J Med Virol. 2020;92:1567-I57I. [CrossRef]

13. Machida M, Nakamura I, Saito R, Nakaya T, Hanibuchi T, Takamiya T, et al. Incorrect use of face masks during the current COVID-19 pandemic among the general public in Japan. Int $J$ Environ Res Public Health. 2020;17(18):6484. [CrossRef]

14. Chen YJ, Qin G, Chen J, et al. Comparison of face-touching behaviors before and during the coronavirus disease 2019 pandemic. JAMA Netw Open. 2020;3:E2016924. [CrossRef]

15. Hung LS. The SARS epidemic in Hong Kong: What lessons have we learned? J R Soc Med. 2003;96:374-378. [CrossRef]

16. Sultanoglu N, Baddal B, Suer K, Sanlidag T. Current situation of covid-19 in northern cyprus. East Mediterr Heal J. 2020;26(6):641645. [CrossRef]

17. Turkish Republic of Northern Cyprus, Ministry of Health. 2020. Available at https://saglik.gov.ct.tr/COVID-19-GENEL-DURUM. Accessed date: 24 November 2020.

18. Turkish Republic of Northern Cyprus Ministry of Health. COVID-19 Announcement. 2020. Available at http://saglik.gov.ct.tr/Haberler/ ArtMID /31973/ArticleID/I29226/Bakan-Pilli-Toplam-373-testyapıldı-pozitif-vaka-yok. Accessed date: I May 2020.

19. van Doremalen N, Bushmaker T, Morris DH, et al. Aerosol and surface stability of SARS-CoV-2 as compared with SARS-CoV-I. N Engl J Med. 2020;382:1564-I567. [CrossRef]

20. Roupa Z, Polychronis G, Latzourakis E, et al. Assessment of knowledge and perceptions of health workers regarding COVID-19: A cross-sectional study from Cyprus. J Community Health. 2021;46:25I-258. [CrossRef]

21. Zhong BL, Luo W, Li HM, Zhang QQ, Liu XG, Li WT, et al. Knowledge, attitudes, and practices towards COVID-19 among Chinese residents during the rapid rise period of the COVID-19 outbreak: a quick online cross-sectional survey. Int J Bio/ Sci. 2020;16(I0):1745-1752. [CrossRef]

22. Khan S, Khan M, Maqsood K, Hussain T, Noor-ul-Huda, Zeeshan M. Is Pakistan prepared for the COVID-19 epidemic? A questionnairebased survey. J Med Virol. 2020;92(7):824-832. [CrossRef]

23. Younis I, Longsheng C, Zulfiqar MI, Imran M, Shah SAA, Hussain M, et al. Regional disparities in Preventive measures of COVID-19 pandemic in China. A study from international students prior knowledge, perception and vulnerabilities. Environ Sci Pollut Res. 2020;28:40355-40370. [CrossRef]

24. Ngwewondo A, Nkengazong L, Ambe LA, et al. Knowledge, attitudes, practices of/towards COVID-19 preventive measures and symptoms: A cross-sectional study during the exponential rise of the outbreak in Cameroon. PLoS Negl Trop Dis. 2020;14:E0008700. [CrossRef]

25. Nnama-Okechukwu CU, Chukwu NE, Nkechukwu CN. COVID-19 in Nigeria: Knowledge and compliance with preventive measures. Soc Work Public Health. 2020;16(3):e0248189. [CrossRef]
26. UI Haq S, Shahbaz P, Boz I. Knowledge, behavior and precautionary measures related to COVID-19 pandemic among the general public of Punjab province, Pakistan. J Infect Dev Ctries. 2020;|4:823-835. [CrossRef]

27. Khaled A, Siddiqua A, Makki S, The knowledge and attitude of the community from the Aseer region, Saudi Arabia, toward COVID19 and their precautionary measures against the disease. Risk Manag Healthc Policy. 2020;13:1825-1834. [CrossRef]

28. Machida M, Nakamura I, Saito R, Nakaya T, Hanibuchi T, Takamiya $\mathrm{T}$, et al. Adoption of personal protective measures by ordinary citizens during the COVID-19 outbreak in Japan. Int $J$ Infect Dis. 2020;94:139-144. [CrossRef]

29. Atchison C, Bowman L, Eaton JW, Imai N, Redd R, Pristera P, et al. Public Response to UK Government Recommendations on COVID19: Population Survey, 17-18 March 2020. Imp Coll London COVID19 Response Team 2020.

30. Kwok KO, Li KK, Chan HHH, Yi YY, Tang A, Wei Wl, et al. Community Responses during Early Phase of COVID-19 Epidemic, Hong Kong. Emerg Infect Dis. 2020;26(7):1575-1579. [CrossRef]

31. Khasawneh Al, Humeidan AA, Alsulaiman JW, Bloukh S, Ramadan $\mathrm{M}$, Al-Shatanawi TN, et al. Medical Students and COVID-19: Knowledge, Attitudes, and Precautionary Measures. A Descriptive Study From Jordan. Front Public Heal. 2020;8:253. [CrossRef]

32. Machida M, Nakamura I, Saito R, Nakaya T, Hanibuchi T, Takamiya $T$, et al. Changes in implementation of personal protective measures by ordinary Japanese citizens: A longitudinal study from the early phase to the community transmission phase of the COVID-19 outbreak. Int J Infect Dis. 2020;96:371-375. [CrossRef]

33. World Health Organization. Advice on the use of masks in the community, during home care and in healthcare settings in the context of the novel coronavirus (2019-nCoV) outbreak [Internet]. 2020. Available at https://apps.who.int/iris/handle/I0665/ 330987. Accessed date: 18 September 2020.

34. World Health Organization. Rational use of personal protective equipment for coronavirus disease 2019 (COVID-19). 2020. Available at https://www.who.int/publications-detail/rational-use-of-per sonal-protective-equipment-for-coronavirus-disease-(covid-19)and-considerations-during-severe-shortages. Accessed date: 26 December 2020.

35. Servick K. Would everyone wearing face masks help us slow the pandemic? Science. (80- ) 2020. [CrossRef]

36. Centers for Disease Prevention and Control. Use of cloth face coverings to help slow the spread of COVID-19. 2020. Available at https:/ / www.cdc.gov/coronavirus/2019-ncov/prevent-gettingsick/diy-cloth-face-coverings.html. Accessed date: 23 February 2021.

37. Sun $C X, H e B, M u D, L I P L, Z H A O H T$, LI ZL, et al. Public Awareness and Mask Usage during the COVID-19 Epidemic: A Survey by China CDC New Media. Biomed Environ Sci. 2020;33:639-645. [CrossRef]

38. Lyu W, Wehby GL. Community use of face masks and COVID-19: Evidence from a natural experiment of state mandates in the US. Health Affairs. 2020;39:1419-1425. [CrossRef]

39. Chaib F. Shortage of personal protective equipment endangering health workers worldwide. WHO. 2020. Available at: https:// www.who.int/news/item/03-03-2020-shortage-of-personalprotective-equipment-endangering-health-workers-worldwide. Accessed date: 10 August 2020.

40. Strzelecki A, Azevedo A, Albuquerque A. Correlation between the spread of COVID-19 and the interest in personal protective measures in Poland and Portugal. Healthcare. 2020;8:203. [CrossRef]

4l. Abdelhafiz AS, Mohammed Z, Ibrahim ME, et al. Knowledge, perceptions, and attitude of Egyptians towards the novel coronavirus disease (COVID-19). J Community Health. 2020;45:881-890. [CrossRef] 